\title{
CONF-9505193-2
}

\section{TRACKING CONTROL OF THE HYDRAULICALLY ACTUATED FLEXIBLE MANIPULATOR *}

D. S. Kwon, S. M. Babcock, B. L. Burks, and R. L. Kress

Robotics \& Process Systems Division

Oak Ridge National Laboratory

Post Office Box 2008

Oak Ridge, Tennessee 37831-6304

\begin{abstract}
The submitted manuscript has been authored by a contractor of the U. S. Government under contract DE-AC05-

84OR21400. Accordingly, the U. S.

Government retains a paid-up, nonexclusive, irrevocable, woridwide license to publish or reproduce the published form of the public, and perform publiciy and display publicly, or allow others to do so, for U. S. Government purposes.
\end{abstract}

To be presented at the 1995 IEEE International Conference on Robotics \& Automation

Nagoya, Japan

May 21-27, 1995

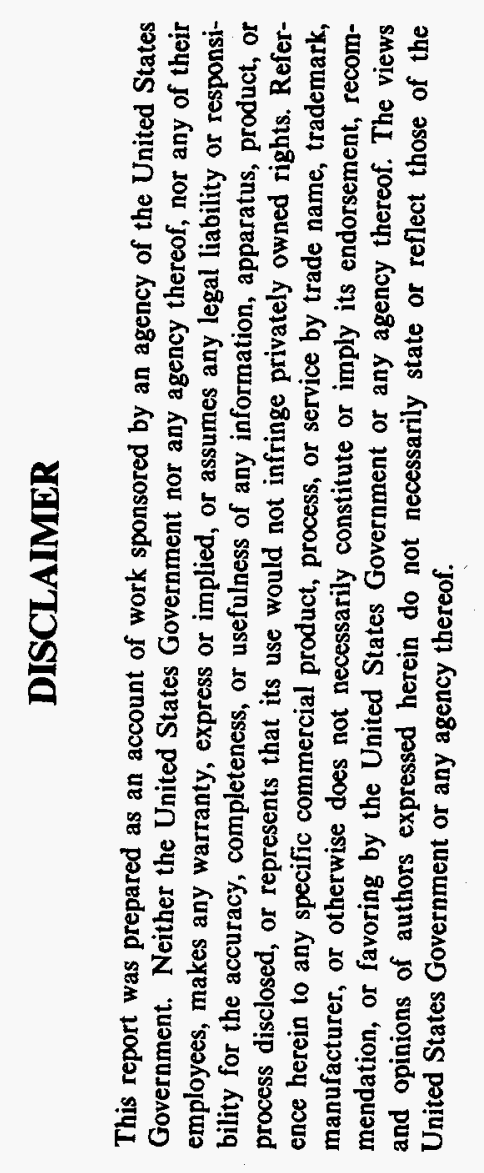

\footnotetext{
* Research sponsored by the Office of Technology Development, U. S. Department of Energy, under contract DEAC05-84OR21400 with Martin Marietta Energy Systems, Inc.
} 


\section{DISCLAIMER}

Portions of this document may be illegible in electronic image products. Images are produced from the best available original document. 


\title{
Tracking Control of the Hydraulically Actuated Flexible Manipulator *
}

\author{
D. S. Kwon, S. M. Babcock, B. L. Burks, and R. L. Kress \\ Robotics \& Process Systems Division \\ Oak Ridge National Laboratory \\ Oak Ridge, TN 37831-6304
}

\begin{abstract}
*
The remediation of single-shell radioactive waste storage tanks is one of the urgent tasks of the Department of Energy that challenge state-of-the-art equipment and methods. The use of long-reach manipulators is being seriously considered for this remediation task. Because of high payload capacity and high length-to-cross-section ratio requirements, these long-reach manipulator systems are expected to use hydraulic actuators and to exhibit significant structural flexibility. The controller has been designed to compensate for the hydraulic actuator dynamics by using a load-compensated velocity feedforward loop and to increase the bandwidth by using a pressure feedback loop. Shaping filter techniques have been applied as a feedforward controller to avoid structural vibrations during operation. Among various types of shaping filter methods investigated, an approach, referred to as a "feedforward simulation filter" that uses embedded simulation, has been presented.
\end{abstract}

\section{INTRODUCTION}

Underground storage tank waste remediation is one of the most urgent tasks among the Environmental Restoration and Waste Management (ER\&WM) Program of the U. S. Department of Energy's (DOE's) Office of Technology Development. The use of long-reach manipulators (LRMs) is being seriously considered as a tank waste retrieval manipulator system (TWRMS), and the prototype test bed is being constructed to test various cleanup scenarios, end-effector tools, and control

\footnotetext{
* Research sponsored by the Office of Technology Development, U.S. Department of Energy, under contract DE-AC05-84OR21400 with Martin Marietta Energy Systems, Inc.
}

schemes. The development of a TWRMS may be one of DOE's most significant robotics projects.

The TWRMS will consist of three elements: an LRM, including a vertical deployment mast, a short-reach dexterous manipulator, and various end-effector tools. From preliminary studies ${ }^{1,2}$ it is anticipated that the LRM will have very low structural natural frequencies and that its structural flexibility will significantly affect the positioning accuracy of the end of the manipulator. Control of the end position of the LRM, considering its flexibility, will be very important to the performance of various cleaning processes with the dexterous manipulator. Because of the heavy weight of the arm and the large payload capacity, the hydraulic actuator is inevitable in spite of the hydraulic oil problem in the radiation environment.

In this research, the control of a large flexible manipulator with a large-capacity hydraulic actuator has been approached in two aspects. One aspect is shaping the command trajectory with filtering methods to avoid the excitation of the resonant frequency of the system. The other aspect is compensating the hydraulic actuator dynamics and the payload effect to achieve good tracking with a large-capacity actuator.

First, many input shaping methods have been proposed to minimize the structural vibration. The prominent filtering methods are impulse shaping filters 3,4 robust notch filters, ${ }^{5}$ and model-based shaping methods. ${ }^{6,7}$ There are many other effective vibration suppression control schemes such as acceleration feedback, ${ }^{8}$ passive damping treatment, ${ }^{9}$ and end-position feedback. ${ }^{10}$ Various approaches are well summarized by Book. ${ }^{11}$ The impulse shaping filter is effective but introduces a tracking delay. ${ }^{12}$ If multiple impulses are used for robust filtering, the increased time delay introduced may be a serious problem for teleoperation and robotic tracking control of a 
very flexible manipulator that has a very low system bandwidth. The robust notch filter method is easy to use and practical. ${ }^{12}$ Since it has a wide filtering band, it is robust to the change of the system dynamics. ${ }^{5}$ However, it also introduces a significant time delay like that of an impulse shaping filter. Both shaping filter methods need at least partial information of the flexible dynamic system (e.g., a dominant vibration frequency or the dominant frequency and damping ratio). The limiting cases of complete knowledge and no knowledge of the structural dynamics are of significant interest. Therefore, two approaches that represent these extremes have been proposed and investigated. ${ }^{12}$ One approach, called the "fuzzy shaping method," does not require precise knowledge of the flexible dynamics. The joint trajectory was modified from the end-position trajectory by fuzzy rules that considered the effect of flexibility to avoid commanding the flexible beam to move like a rigid beam. If we have the knowledge of the dynamic system, the model-based shaping methods such as the inverse dynamic method can be used. The inverse dynamic method guarantees the tracking performance, but it is limited to the off-line trajectory generation because of the noncausal solution for the nonminimum-phase system. Another model-based shaping method, called "feedforward simulation filtering," incorporates the advantages of several other methods: end-position feedback, robust notch filtering, and torque feedforward loop. It requires a complete knowledge of the dynamics of the system, such as that required by the inverse dynamic method, and shows excellent tracking performance.

Second, suppose the filtered joint trajectory has been generated by the shaping filter methods. However, it is not easy to make a large-capacity hydraulic actuator to follow the joint trajectory command. Because of the nonlinear relation between the pressure and the valve opening of the hydraulic actuator, it is very difficult to apply the desired actuator pressure (torque) by adjusting the valve opening. In order to generate desired joint speed regardless of the payload, the valve opening should be adjusted to compensate the load pressure effect. An important issue would be to move the heavy manipulator with heavy payload in the gravitational direction.

This paper presents good tracking results of the feedforward simulation filtering method combined with the load compensated velocity feedforward and pressure feedback method. All results have been generated on the Pacific Northwest Laboratories (PNL) flexible-beam test bed with a real-time control software system called MICA (Modular Integrated Control Architecture). ${ }^{13}$

\section{A. Limitation of shaping methods for the tracking}

Generally speaking, shaping filter methods have focused on not exciting the structural vibration without considering the tracking performance. In end-effector tool applications with the manipulator, the tracking performance is very important. Preshaping the endposition trajectory with filters will determine the filtered desired end-position trajectory and can determine whether the filtered end-position trajectory is acceptable for a specific task. However, the inverse kinematic relation does not guarantee that the joint trajectory does not have the frequency component that was filtered out in multilink cases.

There is another option. From the desired endposition trajectory, the desired joint trajectory is calculated using the inverse kinematic relation. Then, the joint trajectory is filtered by input shaping methods to avoid exciting the structural frequency. This option is very effective, but it does not determine whether the endposition trajectory is acceptable for the task until the manipulator actually moves. This issue has been rarely addressed in literature yet is believed to require resolution in order to use shaping filter methods for the multilink flexible manipulator control. Since the simulation filtering method of this paper minimizes the tracking time-delay, it is expected to produce less tracking error when applied to the multilink cases.

\section{EXPERIMENTAL TEST BED}

To study fundamental control issues associated with structural vibration of the LRM, a test bed was built at PNL. The test bed has a 15-ft-long flexible beam (12 inch height by $3 / 4$ inch width, steel) with a Schilling hydraulic manipulator at the end of the beam, as shown in Fig. 1. The flexible beam represents a simplified LRM dynamically, and the Schilling manipulator represents the dexterous manipulator. An air bearing supports the end of the flexible beam to ensure planar operation. A rack-andpinion style hydraulic rotary actuator (Flo-Tork) has been used as a base actuator. A hydraulic servo valve (Parker ST10-5, $5 \mathrm{gal} / \mathrm{min}$ at $1000 \mathrm{psi}$ ) has been used with a servo valve amplifier (Parker BD90).

\section{MODELING}

\section{A. Flexible beam}

The flexible beam of the PNL test bed was modeled by using the assumed mode method. To obtain an accurate model with a small number of modes, pinnedpinned boundary conditions considering the hub inertia and the end-mass were used for the calculation of mode shape functions. ${ }^{6}$ The test bed was modeled as a single 


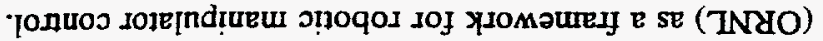

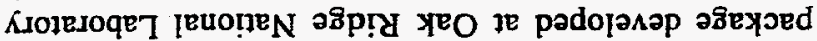

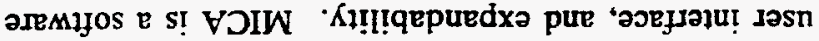

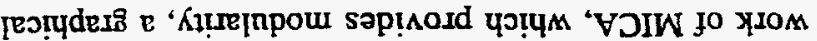

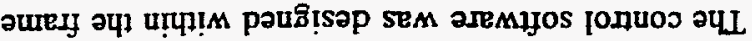

VDWW-aIBAyJOS $\vee \mathrm{V}$

\section{WGISAS TO\&.INOS $A I$}

(9)

$$
\begin{aligned}
& { }^{b_{L G}}+X D=X \\
& b_{L g}+X V=X
\end{aligned}
$$

woj uoṇenbo

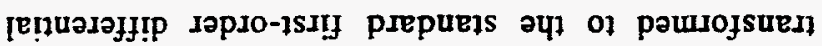

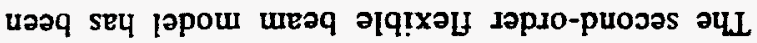

$$
{ }^{2} a^{7} d=b_{l}
$$

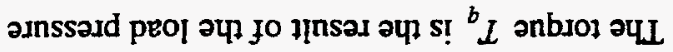

'pṃny

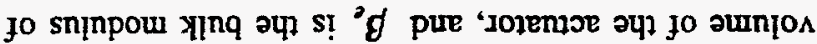

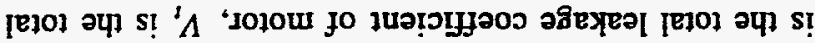

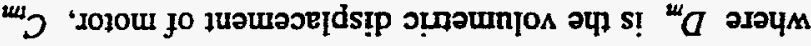

(

$$
{ }^{7} d \frac{{ }^{2} g_{t}}{{ }^{2} \Lambda}+{ }^{7} d^{u w} \partial+\Omega^{w} a={ }^{7} \delta
$$

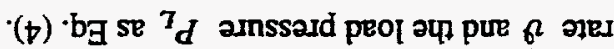

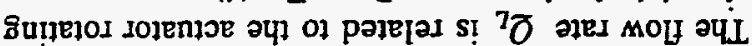

-8utuado әn[en әफ s! " $x$

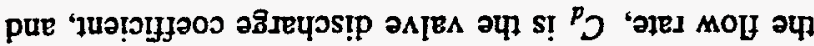

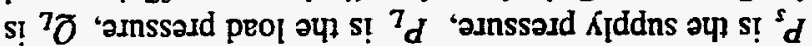

( $)$

$$
\left({ }^{7} d\left({ }^{n} x\right) u 8 ! s-{ }^{s} d\right) u 81 s_{*}
$$

$$
\left({ }^{7} d\left({ }^{A} x\right) u 8 \underline{1} s-{ }^{s} d\right) s q p \Lambda^{\wedge} x^{p} \partial={ }^{7} \partial
$$

$$
{ }^{7} d-{ }^{s} d{ }^{A} x^{p} \partial={ }^{7} \partial
$$

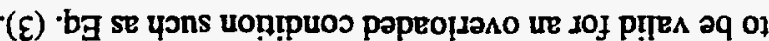

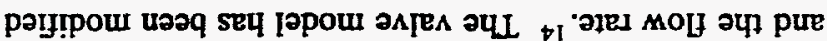

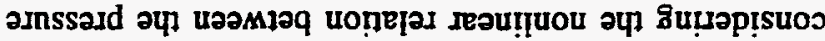

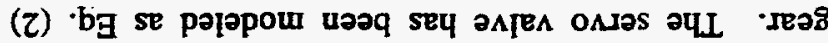

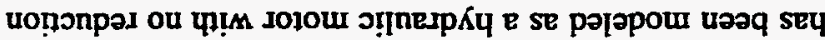

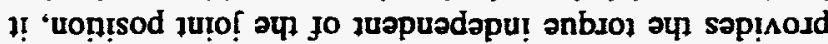

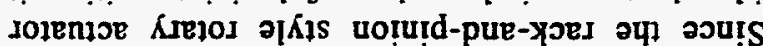

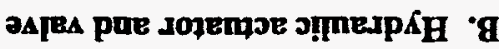

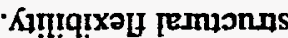

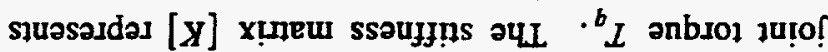

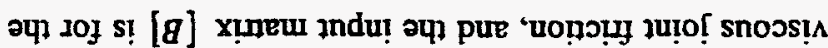

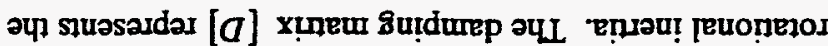
pue sseu pue ue pue 'ethou! peuonezos qny e 'suoṇouny

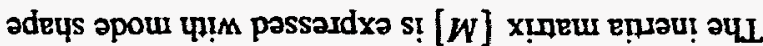

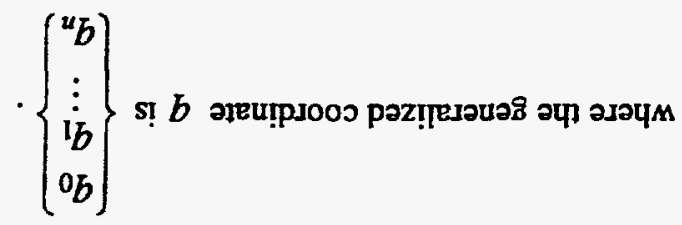

$$
{ }^{b} L[g]=b[X]+b[a]+b[W]
$$

4!บ:M

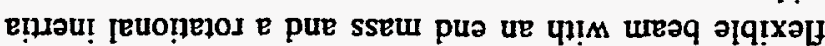

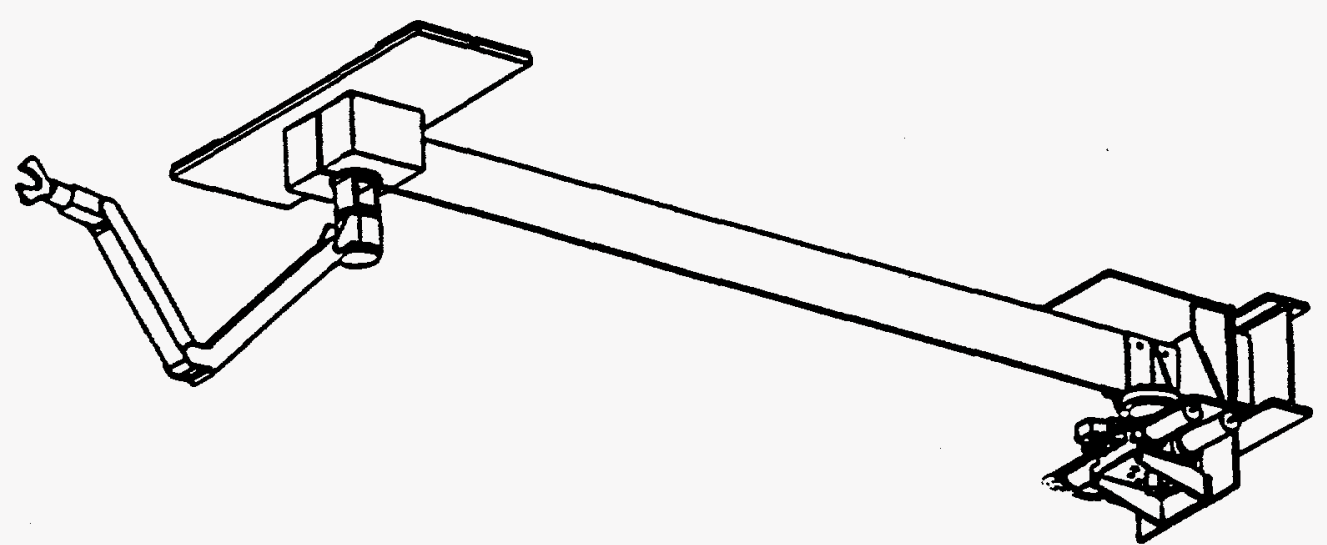


MICA yields operational codes that are portable among different manipulators and operating environments. It allows precise operation of multiple processors that have to be coordinated to control manipulators. Within the MICA framework, specific aspects of the LRM control have been considered during the controller development stage.

\section{B. Hardware-VME System}

The hardware for the control system consists of a SUN workstation and a VME bus-based system rack, as shown in Fig. 2. The SUN workstation is used for the graphical user interface and for a supervisor of the control system. The control system rack contains central processing unit (CPU) boards and several interface cards for data acquisition. Depending on the computational load, CPU boards can be added and the control software can be adapted easily for multiple processors. Data exchange between the SUN workstation and the system rack is by Ethernet.

\section{CONTROL ALGORITHM}

\section{A. Filtering methods}

The feedforward simulation filter is used as an alternative to the inverse dynamic method. The feedforward simulation filtering method using the knowledge of the dynamics gives good tracking performance with the minimum time delay. As Cannon and Schmitz ${ }^{10}$ indicated, end-position feedback could provide a much higher closed-loop bandwidth (beyond the clamped natural frequency) than that of a joint-based closed-loop feedback system. However, end-position feedback is very sensitive to parameter variation and modeling error. It may not be appropriate for practical applications with dynamic system information that are approximately known. The conventional proportionalderivative (PD) joint feedback system usually yields good stability, but the closed-loop bandwidth cannot be greater than the clamped natural frequency. In practical applications, it is usually less than half the fundamental clamped natural frequency. ${ }^{11}$

Figure 3 describes a feedforward simulation filtering method that integrates most of the advantages of the above methods. Since the higher bandwidth system has less time delay with the shaping filter, the closed-loop system, which has two or three times higher bandwidth than that of the joint feedback loop, was made with the end-position feedback, including joint rate feedback. A feedforward torque loop was added to improve tracking. As mentioned above, because end-position feedback is conditionally stable and sensitive to the modeling errors, it may be difficult to use for actual applications. Therefore, the end-position feedback with a robust notch shaping filter was used in the simulation to generate a joint trajectory that makes the end position follow the desired filtered trajectory. Since the appropriate joint trajectory was generated, the joint PD controller, even with low gain, gives good tracking performance of the end position, as shown in Fig. 4.

\section{B. Load-compensated feedforward control}

Since the desired joint trajectory has been generated by using the shaping filter, the next important step is how to make the hydraulic actuator follow the desired trajectory precisely, and how to apply the desired torque. Because of the nonlinear relation between the pressure and the valve opening of the hydraulic actuator, it is very difficult to apply the desired actuator pressure (torque) by adjusting the valve openings. Therefore, the desired joint velocity has been applied as a feedforward command.

First, the required flow rate $Q_{L d}$ has been calculated from the desired joint velocity $\dot{\vartheta}_{d}$ and the measured load pressure.

$Q_{L d}=D_{m} \dot{\vartheta}_{d}+C_{t m} P_{L}+\frac{V_{t}}{4 \beta_{e}} \dot{P}_{L}$

Second, the desired valve opening $x_{y d}$ has been calculated by using the measured load pressure and the calculated required flow-rate:

$$
x_{v d}=\frac{Q_{L d}}{C_{d} \sqrt{P_{s}-P_{L}}}
$$

Then, the desired valve opening has been applied as a feedforward control. Since the load pressure is measured, the desired torque (converted to pressure) and the measured load pressure have been applied as an outer pressure feedback loop. This pressure feedback loop not only increases the stability of the feedback controller, but also improves the tracking performance. The final input command to the servo valve is the sum of the feedforward command and the feedback control signal.

$$
x_{v}=x_{v d}+K_{p}\left(\theta_{f d}-\theta\right)+K_{v}\left(\dot{\theta}_{f d}-\dot{\theta}\right)+K_{p r}\left(P_{L d}-P_{L}\right)
$$

\section{EXPERIMENTAL RESULTS : FEEDFORWARD SIMULATION FILTER METHODS}

As shown in Fig. 4, the original end-position desired trajectory has been modified by the robust notch filter, 


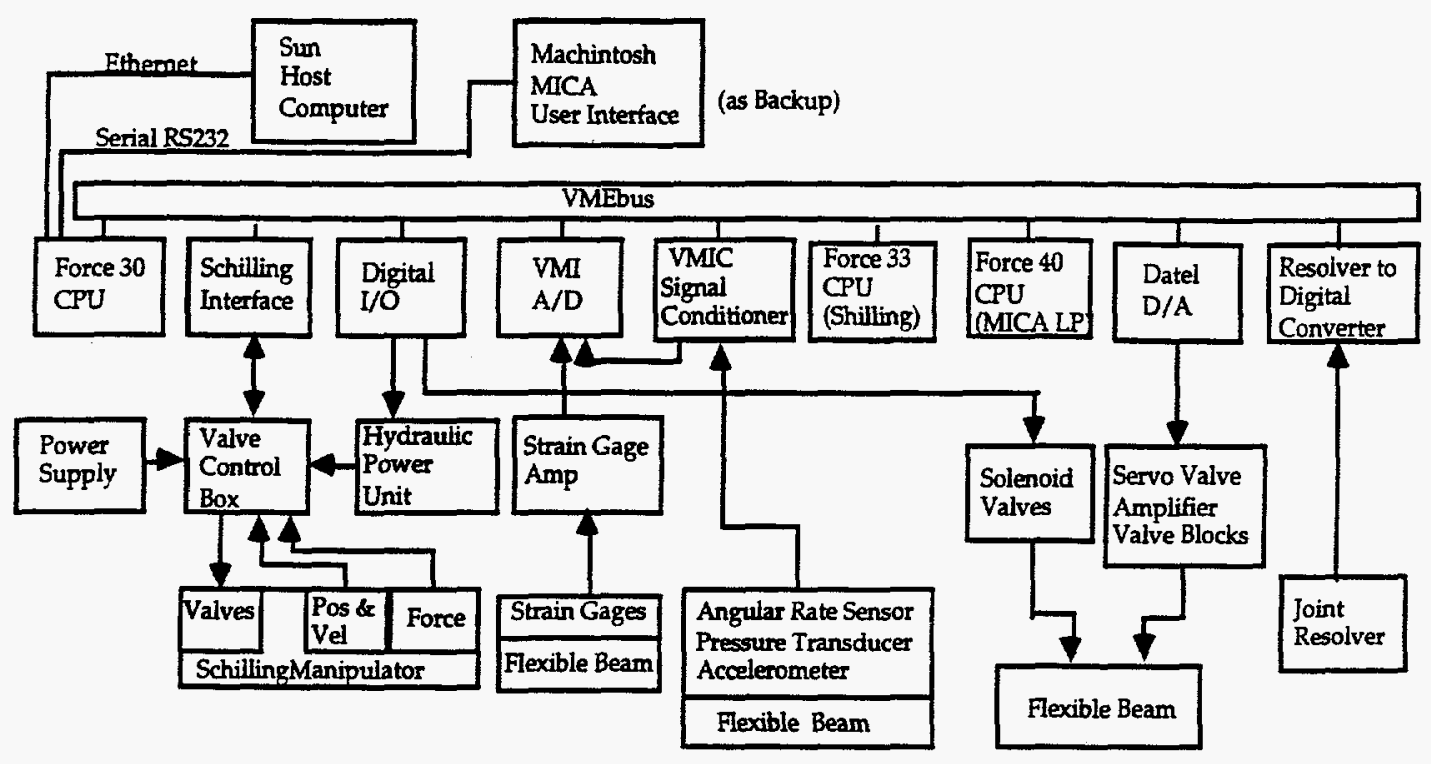

Fig. 2. VME system controller for the flexible-beam test bed.

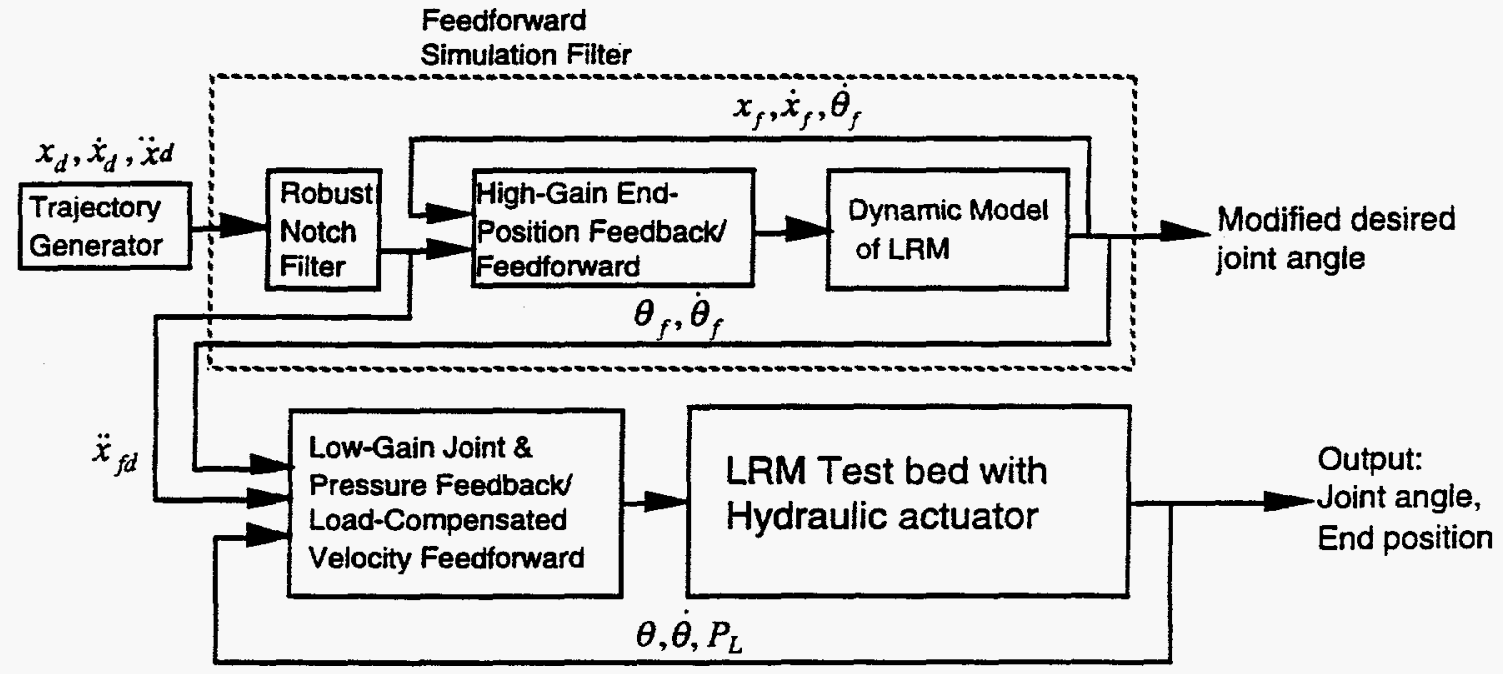

Fig. 3. The feedforward simulation filtering method. 
(a) Joint trajectory

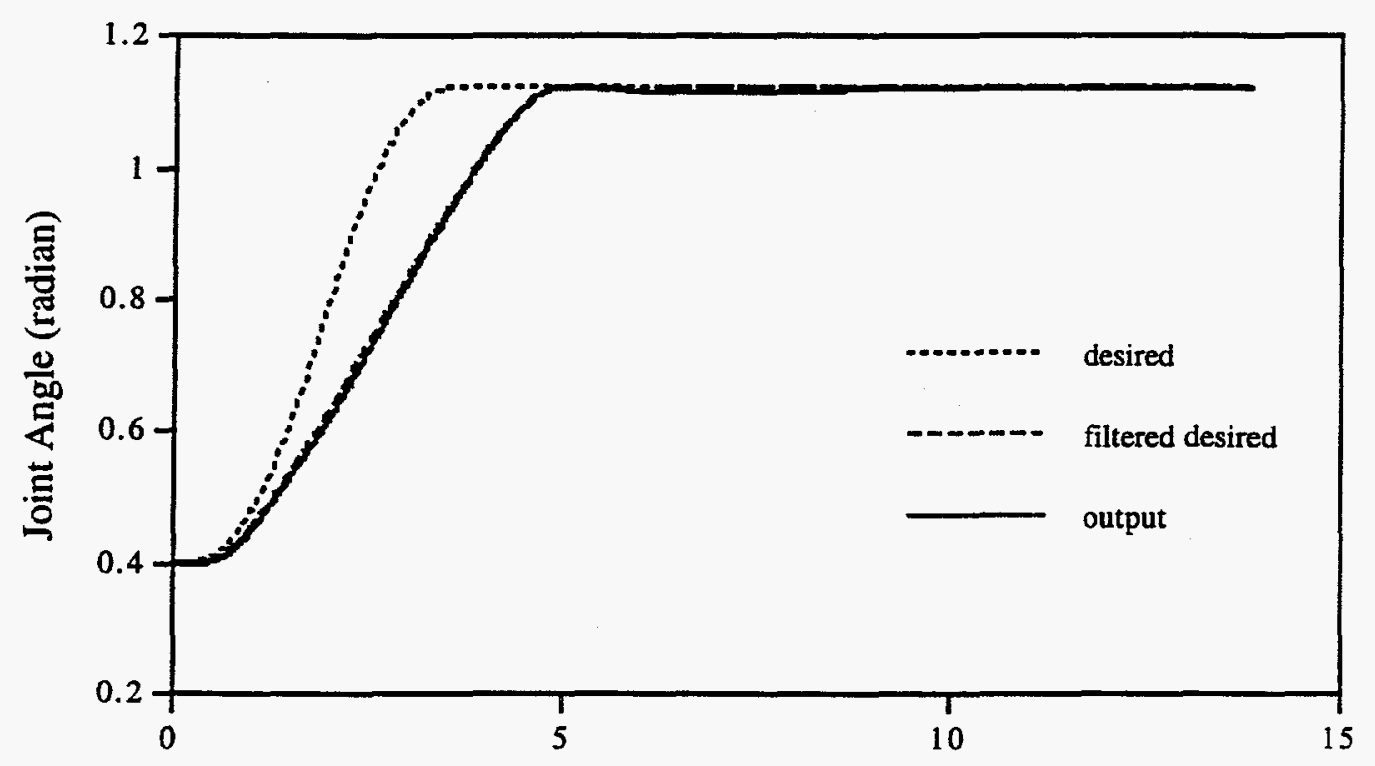

Time (second)

(b) End-position trajectory

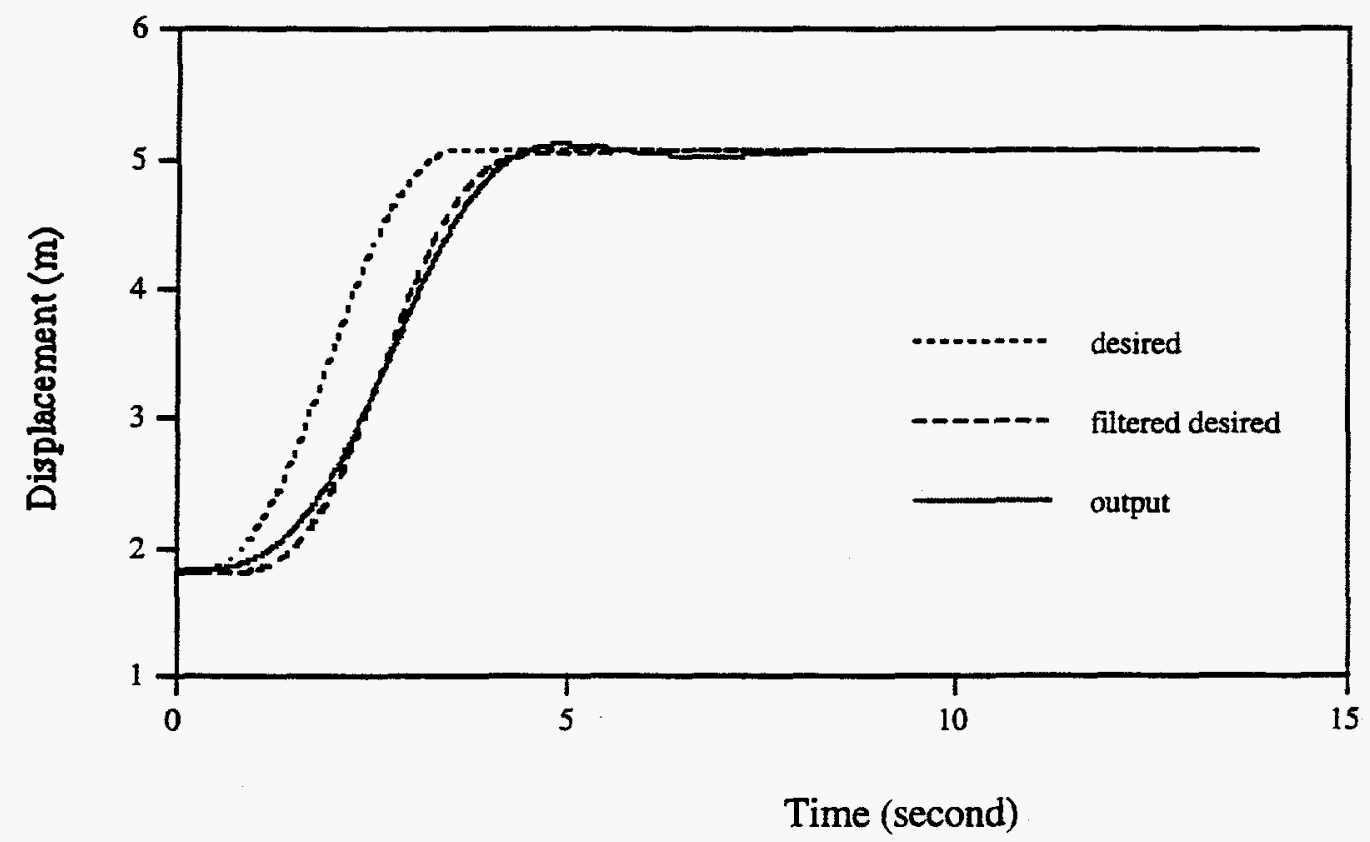

Fig. 4. Experimental results of the feedforward simulation filtering method. 
which is tuned for the high-bandwidth end-position feedback simulation model. The filtered end-position trajectory is given as an input to the end-position feedback simulation model. Then, the joint angle and velocity output of the simulation are the truly filtered trajectories considering the flexible dynamics. If the same torque that was used in the simulation system is applied to the real system and the joint position of the real system is exactly tracking the joint output of the simulation system, the end position of the real system can be assumed to follow the end-position output of the simulation system. The experimental results show predicted good tracking without overshoot. With the low-gain joint PD controller, we could obtain the tracking performance of the highgain, high-bandwidth end-position feedback controller. This is the valuable advantage of the feedforward simulation filter.

\section{CONCLUSIONS}

The feedforward simulation method with the load compensation gives almost perfect tracking performance at the price of the knowledge of the dynamics and calculation burden. Therefore, the trade-off between the performance and the requirement for prior knowledge of the system and the calculation burden should be considered in the control system design. The load compensated velocity feedforward and pressure feedback control method enhanced tracking performance of the hydraulic actuator. This method requires only the hydraulic servo valve model information, and it would be useful for any hydraulic actuator system controls. ORNL is pursuing extension of the shaping filter methods to actual three-dimensional, multilink LRMs. The use of a real-time fast Fourier transform to adapt the shaping filter is being tested for situations when variations in the manipulator configuration or payload result in significant changes in the fundamental natural frequency of the system's structure.

\section{REFERENCES}

1. D. S. KWON, S. MARCH-LEUBA, S. M. BABCOCK, and W. R. HAMEL, "Parametric Design Studies of Long-Reach Manipulators," Proceedings of the American Nuclear Society Fifth Topical Meeting on Robotics and Remote Handling, pp. 265-73, April 1993.

2. D. S. KWON, S. MARCH-LEUBA, S. M. BABCOCK, and W. R. HAMEL, Key Design Requirements for Long-Reach Manipulators, ORNL/TM-12251, Oak Ridge National Laboratory, September 1993.
3. N. C. SINGER and W. P. SEERING, "Preshaping Command Inputs to Reduce System Vibration," Trans. ASME, Journal of Dynamic Systems, Measurement, and Control, Vol. 112, pp. 76-82, 1990.

4. D. P. MAGEE and W. J. BOOK, "The Application of Input Shaping to a System with Varying Parameters," Proceedings of ASME Japan-USA Symposium on Flexible Automation, San Francisco, Vol. 1, pp. 519-26, 1992.

5. J. F. JANSEN, Control and Analysis of a SingleLink Flexible Beam with Experimental Verification, ORNL/TM-12198, Oak Ridge National Laboratory, December 1992.

6. D. S. KWON and W. J. BOOK, "A Time Domain Inverse Dynamic Method for the End-Point Tracking Control of a Single-Link Flexible Manipulator," the transaction of ASME, Journal of Dynamic Systems, Measurement, and Control, Vol. 116, pp. 193-200, June, 1994.

7. E. BAYO and B. PADEN, "On Trajectory Generation for Flexible Robots," Journal of Robotic Systems, Vol. 4, No. 2, pp. 229-35, 1987.

8. P. KOTNIK, S. YURKOVICH, and Ü. ÖZG ÜNER, "Acceleration Feedback for Control of a Flexible Manipulator Arm," Journal of Robotic Systems, Vol. 5, No. 3, pp. 181-96, 1988.

9. T. E. ALBERTS, H. XIA, and Y. CHEN, "Dynamic Analysis to Evaluate Viscoelastic Passive Damping Augmentation for Space Shuttle Remote Manipulator System," Trans. ASME, Journal of Dynamic Systems, Measurement, and Control, Vol. 114, pp. 468-75, 1992.

10. R. H. CANNON and E. SCHMITZ, "Initial Experiments on the End-Point Control of a Flexible OneLink Robot," International Journal of Robotic Research, Vol. 3, No. 3, pp. 62-75, Fall 1984.

11. W. J. BOOK, "Controlled Motion in an Elastic World," Trans. ASME, Journal of Dynamic Systems, Measurement, and Control, Vol. 115, pp. 252-61, June 1993.

12. D. S. KWON, D. H. HWANG, S. M. BABCOCK, and B. L. BURKS, "Input Shaping Filter Methods for the Control of Structurally Flexible, Long-Reach Manipulators," Proceedings of 1994 IEEE International 
Conference on Robotics and Automation, pp. 3259-64, San Diego, CA, May 8-13, 1994.

13. P. L. BUTLER, "An Integrated Architecture for Modular Control Systems," Robotics and Autonomous System, Vol. 10, No. 2-3, 1992.
14. H. E. MERRITT, Hydraulic Control Systems, John Wiley \& Sons, Inc., 1967. 